\title{
Existence of Positive Solutions for Fourth-Order Boundary Value Problems with Sign-Changing Nonlinear Terms
}

\author{
Xingfang Feng and Hanying Feng \\ Department of Mathematics, Shijiazhuang Mechanical Engineering College, Shijiazhuang, Hebei 050003, China \\ Correspondence should be addressed to Hanying Feng; fhanying@126.com
}

Received 26 June 2013; Accepted 2 October 2013

Academic Editors: D. D. Hai and L. Wang

Copyright (C) 2013 X. Feng and H. Feng. This is an open access article distributed under the Creative Commons Attribution License, which permits unrestricted use, distribution, and reproduction in any medium, provided the original work is properly cited.

the existence of positive solutions for a fourth-order boundary value problem with a sign-changing nonlinear term is investigated. By using Krasnoselskii's fixed point theorem, sufficient conditions that guarantee the existence of at least one positive solution are obtained. An example is presented to illustrate the application of our main results.

\section{Introduction}

In this paper, we consider the existence of positive solutions to the following fourth-order boundary value problem (BVP):

$$
\begin{gathered}
u^{(4)}(t)-\lambda[f(t, u)+p(t)]=0, \quad \text { a.e. } t \in(0,1), \\
u(0)=u(1)=u^{\prime \prime}(0)=u^{\prime \prime}(1)=0,
\end{gathered}
$$

where $\lambda$ is a positive parameter, $f:(0,1) \times[0, \infty) \rightarrow[0, \infty)$ is continuous and may be singular at $t=0,1$, and $p$ : $(0,1) \rightarrow(-\infty, \infty)$ is Lebesgue integrable and has finitely many singularities in $[0,1]$.

Boundary value problems for ordinary differential equations play a very important role in both theory and applications. They are used to describe a large number of physical, biological, and chemical phenomena. The work of Timoshenko [1] on elasticity, the monograph by Soedel [2] on deformation of structures, and the work of Dulcska [3] on the effects of soil settlement are rich sources of such applications. There has been a great deal of research work on BVPs for second and higher order differential equations, and we cite as recent contributions the papers of Anderson and Davis [4], Baxley and Haywood [5], and Hao et al. [6]. For surveys of known results and additional references, we refer the readers to the monographs by Agarwal et al. $[7,8]$.

Many authors have studied the existence of positive solutions for fourth-order boundary value problems where the nonlinearity takes nonnegative values, see [9-13]. However, for problems with sign-changing nonlinearities, only a few studies have been reported.

Owing to the importance of high order differential equations in physics, the existence and multiplicity of the solutions to such problems have been studied by many authors, see [9, 12-17]. They obtained the existence of positive solutions provided $f$ is superlinear or sublinear in $u$ by employing the cone expansion-compression fixed point theorem.

In [18], by using the strongly monotone operator principle and the critical point theory to discuss BVP

$$
\begin{aligned}
& u^{(4)}(t)-f(t, u)=0, \quad t \in[0,1] \\
& u(0)=u(1)=u^{\prime \prime}(0)=u^{\prime \prime}(1)=0
\end{aligned}
$$

the authors established some sufficient conditions for $f$ to guarantee that the problem has a unique solution, at least one nonzero solution, or infinitely many solutions.

In [10], Feng and Ge considered the fourth-order singular differential equation

$$
u^{(4)}(t)-\lambda g(t) f(u(t))=0, \quad t \in(0,1),
$$

subject to one of the following boundary conditions:

$$
\begin{gathered}
u(0)=u(1)=u^{\prime \prime}(0)=u^{\prime \prime}(1)=0, \\
u(0)=u^{\prime}(1)=u^{\prime \prime}(0)=u^{\prime \prime \prime}(1)=0,
\end{gathered}
$$

where $\lambda>0$. By using a fixed point index theorem in cones and the upper and lower solutions method, the authors 
discussed the existence of positive solutions for the above BVP.

However, most papers only focus on attention to the case where the nonlinearity has no singularities or/and takes nonnegative values on $[0,1]$ and $p(t)=0$. Inspired by the work of the above papers, our aim in the present paper is to investigated the existence of positive solutions to BVP (1) by employing the fixed point theorem of cone expansion and compression of norm type. Some well-known results in the literature are generalized and improved.

By singularity we mean that the function $f(t, u), p(t)$ in BVP (1) are allowed to be unbounded at some point. In the paper, BVP (1) is allowed to have finitely many singularities in $[0,1]$. In $\operatorname{BVP}(1), p(t)$ are allowed to change sign and tend to negative infinity. An element $u \in C[0,1]$ for a.e. $t \in[0,1]$ is called a positive solution of BVP (1) if it satisfies BVP (1) and $u(t) \geq 0$ for any $t \in[0,1]$.

\section{Preliminaries and Several Important Lemmas}

Let $E=C[0,1]$ be equipped with norm $\|u\|=\max _{t \in[0,1]}|u(t)|$, then $(E,\|\cdot\|)$ is a real Banach space.

Definition 1. We define a ordering in $E$ by $u \preccurlyeq v$ for $u, v \in E$ if and only if

$$
u(t) \leq v(t), \quad t \in[0,1]
$$

In the following, let us define a cone $P$ in $E$ by

$$
P=\{u \in E: u \geq G(t, t) \cdot\|u\| \text { for } t \in[0,1]\},
$$

where

$$
G(t, s)= \begin{cases}s(1-t), & 0 \leq s \leq t \leq 1 \\ t(1-s), & 0 \leq t \leq s \leq 1\end{cases}
$$

is the Green's function of the following BVP

$$
\begin{gathered}
-u^{\prime \prime}(t)=0, \quad t \in(0,1), \\
u(0)=u(1)=0 .
\end{gathered}
$$

Obviously

$$
G(t, s) \leq G(t, t), \quad G(t, s) \leq G(s, s), \quad G(t, s) \leq \frac{1}{4},
$$$$
\text { for any } t \in[0,1], s \in[0,1] \text {. }
$$

For convenience, we list the following assumptions:

$\left(H_{1}\right) f:(0,1) \times[0, \infty) \rightarrow[0, \infty)$ is continuous and there exist constants $\alpha, \beta, 0<\alpha<\beta<+\infty$, such that for any $(t, u) \in(0,1) \times[0, \infty)$,

$$
c^{\beta} f(t, u) \leq f(t, c u) \leq c^{\alpha} f(t, u) \quad \text { if } 0 \leq c \leq 1,
$$

$\left(H_{2}\right) p:(0,1) \rightarrow(-\infty, \infty)$ is Lebesgue integrable such that

$$
\begin{gathered}
0<\int_{0}^{1} p_{-}(s) \mathrm{d} s=r<+\infty, \\
0<\int_{0}^{1} G(s, s)\left[f(s, 1)+p_{+}(s)\right] \mathrm{d} s=l<+\infty,
\end{gathered}
$$

where $p_{+}(s)=\max \{p(s), 0\}, p_{-}(s)=\max \{-p(s), 0\}$.

Remark 2. The inequality (10) is equivalent to the following inequality:

$$
c^{\alpha} f(t, u) \leq f(t, c u) \leq c^{\beta} f(t, u) \quad \text { if } c \geq 1 .
$$

For any $x \in C[0,1]$, let us define a function $[\cdot]^{*}$ by

$$
[x(t)]^{*}= \begin{cases}x(t), & x(t) \geq 0, \\ 0, & x(t)<0 .\end{cases}
$$

Let $w(t)=\int_{0}^{1} \mathrm{~d} s \int_{0}^{1} \lambda G(t, s) G(s, \tau) p_{-}(\tau) \mathrm{d} \tau, t \in[0,1]$. Obviously $w(t)$ is continuous on $[0,1]$. By $\left(H_{2}\right)$, we obtain

$$
\begin{aligned}
0<w(t) & =\int_{0}^{1} \mathrm{~d} s \int_{0}^{1} \lambda G(t, s) G(s, \tau) p_{-}(\tau) \mathrm{d} \tau \\
& \leq \frac{1}{16} \lambda \int_{0}^{1} p_{-}(\tau) \mathrm{d} \tau<+\infty,
\end{aligned}
$$

so $w(t)$ is well defined in $E$. By direct computation, we have

$$
\begin{aligned}
& w^{(4)}(t)-\lambda p_{-}(t)=0, \quad \text { a.e. } t \in(0,1) \\
& w(0)=w(1)=w^{\prime \prime}(0)=w^{\prime \prime}(1)=0
\end{aligned}
$$

which imply that $w(t)$ is a positive solutions of the following BVP:

$$
\begin{gathered}
u^{(4)}(t)-\lambda p_{-}(t)=0, \quad \text { a.e. } t \in(0,1) \\
u(0)=u(1)=u^{\prime \prime}(0)=u^{\prime \prime}(1)=0
\end{gathered}
$$

Now, we consider the following BVP:

$$
\begin{gathered}
u^{(4)}(t)-\lambda\left[f\left(t,[u(t)-w(t)]^{*}\right)+p_{+}(t)\right]=0, \quad \text { a.e. } t \in(0,1), \\
u(0)=u(1)=u^{\prime \prime}(0)=u^{\prime \prime}(1)=0 .
\end{gathered}
$$

It is well known that $u \in C[0,1]$ for a.e. $t \in(0,1)$ is a solution of BVP (17) if and only if $u$ is a solution of the following nonlinear integral equation:

$$
\begin{aligned}
u(t)=\int_{0}^{1} \mathrm{~d} s \int_{0}^{1} \lambda G(t, s) G(s, \tau) & {\left[f\left(\tau,[u(\tau)-w(\tau)]^{*}\right)\right.} \\
+ & \left.p_{+}(\tau)\right] \mathrm{d} \tau, \quad t \in[0,1] .
\end{aligned}
$$


Define an operator $T: E \rightarrow E$ as

$$
\begin{aligned}
T u(t)=\int_{0}^{1} \mathrm{~d} s \int_{0}^{1} \lambda G(t, s) G(s, \tau)[ & f\left(\tau,[u(\tau)-w(\tau)]^{*}\right) \\
& \left.+p_{+}(\tau)\right] \mathrm{d} \tau, \quad t \in[0,1] .
\end{aligned}
$$

Obviously, the existence of solutions of the BVP (17) is equivalent to the existence of fixed points of the operator $T$ in the real Banach space $E$.

Lemma 3. Suppose that $\left(H_{1}\right)$ holds, then $f(t, u)$ is nondecreasing in $u$ in $[0,+\infty)$, for any fixed $t \in(0,1)$.

Proof. For any fixed $t \in(0,1)$ and for any $u_{1}, u_{2} \in[0,+\infty)$, without the loss of the generality, let $0 \leq u_{1} \leq u_{2}$. If $u_{2}=0$, obviously the equation $f\left(t, u_{1}\right)=f\left(t, u_{2}\right)=f(t, 0)$ holds. If $u_{2} \neq 0$, let $c_{0}=u_{1} / u_{2}$, then we obtain $0 \leq c_{0} \leq 1$. It follows from (10) that

$$
f\left(t, u_{1}\right)=f\left(t, c_{0} u_{2}\right) \leq c_{0}^{\alpha} f\left(t, u_{2}\right) \leq f\left(t, u_{2}\right),
$$

that is, $f(t, u)$ is nondecreasing in $u$ in $[0,+\infty)$.

Lemma 4. If $v$ with $w \leq v$ is a positive solution of the BVP (17), then $v-w$ is a positive solution of BVP (1).

Proof. Assume that $v$ is a positive solution of BVP (17) such that $w \leq v$, then from (17) and the definition of $[\cdot]^{*}$, we have

$$
\begin{aligned}
v^{(4)}(t)-\lambda[ & f\left(t,[v(t)-w(t)]^{*}\right) \\
& \left.+p_{+}(t)\right]=0, \quad \text { a.e. } t \in(0,1), \\
v(0)= & v(1)=v^{\prime \prime}(0)=v^{\prime \prime}(1)=0 .
\end{aligned}
$$

Let $x(t)=v(t)-w(t)$, then $x^{(4)}(t)=v^{(4)}(t)-w^{(4)}(t)$ for a.e. $t \in(0,1)$, which imply that

$$
v^{(4)}(t)=x^{(4)}(t)+\lambda p_{-}(t) \quad \text { for a.e. } t \in(0,1) .
$$

Thus, (21) becomes

$$
\begin{gathered}
x^{(4)}(t)-\lambda\left[f(t, x(t))+p_{+}(t)-p_{-}(t)\right]=0, \\
\text { a.e. } t \in(0,1), \\
x(0)=x(1)=x^{\prime \prime}(0)=x^{\prime \prime}(1)=0 .
\end{gathered}
$$

Notice that $p(t)=p_{+}(t)-p_{-}(t)$ and (23), we know that $x(t)$ is a positive solution of BVP (1), that is, $v-w$ is a positive solution of BVP (1).

Lemma 5. Assume that $\left(H_{1}\right)$ and $\left(H_{2}\right)$ hold. Then, $T: P \rightarrow P$ is well defined and $T: P \rightarrow P$ is a completely continuous operator.
Proof. For any $u \in P$, choose $0<\sigma<1$ such that $\sigma\|u\|<1$, then we obtain $\sigma[u(t)-w(t)]^{*} \leq \sigma u(t) \leq \sigma\|u\|<1$. Thus, by (10), (12), and Lemma 3, we have

$$
\begin{aligned}
f\left(t,[u(t)-w(t)]^{*}\right) & =f\left(t, \frac{1}{\sigma} \cdot \sigma[u(t)-w(t)]^{*}\right) \\
& \leq\left(\frac{1}{\sigma}\right)^{\beta} f\left(t, \sigma[u(t)-w(t)]^{*}\right) \\
& \leq \sigma^{-\beta} f(t, \sigma\|u\|) \\
& \leq \sigma^{\alpha-\beta}\|u\|^{\alpha} f(t, 1) .
\end{aligned}
$$

Hence, for any $t \in[0,1]$, we get

$$
\begin{aligned}
& T u(t)=\int_{0}^{1} \mathrm{~d} s \int_{0}^{1} \lambda G(t, s) G(s, \tau)[ f\left(\tau,[u(\tau)-w(\tau)]^{*}\right) \\
&\left.+p_{+}(\tau)\right] \mathrm{d} \tau
\end{aligned}
$$

where $K_{1}=(1 / 4) \lambda \cdot \max \left\{\sigma^{\alpha-\beta}\|u\|^{\alpha}, 1\right\}$. Thus, $T: P \rightarrow E$ is well defined.

Next, for any $u \in P$, let $v(t)=T u(t)$. Then, there exists $t_{0} \in[0,1]$ such that $\|v\|=v\left(t_{0}\right)$. Since

$$
\begin{aligned}
& \frac{G(t, s)}{G\left(t_{0}, s\right)} \\
& =\left\{\begin{aligned}
\frac{s(1-t)}{s\left(1-t_{0}\right)} & =\frac{1-t}{1-t_{0}} & & \\
& \geq 1-t \geq t(1-t), & & s \leq t_{0}, t, \\
\frac{s(1-t)}{t_{0}(1-s)} & \geq \frac{s(1-t)}{s(1-s)}=\frac{1-t}{1-s} & & \\
& \geq 1-t \geq t(1-t), & & t_{0} \leq s \leq t, \\
\frac{t(1-s)}{s\left(1-t_{0}\right)} & \geq \frac{t\left(1-t_{0}\right)}{s\left(1-t_{0}\right)}=\frac{t}{s} & & \\
& \geq t \geq t(1-t), & & t \leq s \leq t_{0}, \\
\frac{t(1-s)}{t_{0}(1-s)} & =\frac{t}{t_{0}} \geq t \geq t(1-t), & & t, t_{0} \leq s,
\end{aligned}\right.
\end{aligned}
$$

we obtain

$$
G(t, s) \geq G(t, t) G\left(t_{0}, s\right) .
$$


Hence, we have

$$
\begin{gathered}
v(t)=\int_{0}^{1} \mathrm{~d} s \int_{0}^{1} \lambda G(t, s) G(s, \tau)\left[f\left(\tau,[u(\tau)-w(\tau)]^{*}\right)\right. \\
\left.+p_{+}(\tau)\right] \mathrm{d} \tau \\
\geq \int_{0}^{1} \mathrm{~d} s \int_{0}^{1} \lambda G\left(t_{0}, s\right) G(t, t) G(s, \tau) \\
\quad \times\left[f\left(\tau,[u(\tau)-w(\tau)]^{*}\right)+p_{+}(\tau)\right] \mathrm{d} \tau \\
=G(t, t) v\left(t_{0}\right)=G(t, t)\|v\|, \quad t \in[0,1]
\end{gathered}
$$

So, we conclude that $T P \subset P$.

Let $D \subset P$ be any bounded set, then there exists a constant $L>0$ such that $\|u\| \leq L$ for any $u \in D, t \in[0,1]$, we have

$$
[u(t)-w(t)]^{*} \leq u(t) \leq\|u\| \leq L \leq L+1 .
$$

By (12), (29) and Lemma 3, for any $u \in D, t \in[0,1]$, we have

$$
f\left(t,[u(t)-w(t)]^{*}\right) \leq f(t, L+1) \leq(L+1)^{\beta} f(t, 1) .
$$

From (9), (30), $\left(H_{2}\right)$ and Lemma 3, for any $u \in D$, we have

$$
\begin{array}{r}
T u(t)=\int_{0}^{1} \mathrm{~d} s \int_{0}^{1} \lambda G(t, s) G(s, \tau)\left[f\left(\tau,[u(\tau)-w(\tau)]^{*}\right)\right. \\
\left.+p_{+}(\tau)\right] \mathrm{d} \tau \\
\leq \int_{0}^{1} \mathrm{~d} s \int_{0}^{1} \lambda G(t, t) G(\tau, \tau)\left[f\left(\tau,[u(\tau)-w(\tau)]^{*}\right)\right. \\
\left.+p_{+}(\tau)\right] \mathrm{d} \tau
\end{array}
$$

where $K_{2}=(1 / 4) \lambda \cdot \max \left\{(L+1)^{\beta}, 1\right\}$. Therefore, $T(D)$ is uniformly bounded.

Since $G(t, s)$ is continuous in $[0,1] \times[0,1], G(t, s)$ is uniformly continuous. Hence, for any $\varepsilon>0$, there exists $\delta>0$ such that $\left|t_{1}-t_{2}\right|<\delta$, for any $s \in[0,1]$ we have

$$
\left|G\left(t_{1}, s\right)-G\left(t_{2}, s\right)\right| \leq \varepsilon\left(\lambda \max \left\{(L+1)^{\beta}, 1\right\} l\right)^{-1}
$$

from (30), (32) and $\left(H_{2}\right)$, for any $u \in D$, we obtain

$$
\begin{aligned}
& \left|T u\left(t_{1}\right)-T u\left(t_{2}\right)\right| \\
& =\mid \int_{0}^{1} \lambda\left(G\left(t_{1}, s\right)-G\left(t_{2}, s\right)\right) \mathrm{d} s \\
& \quad \times \int_{0}^{1} G(s, \tau)\left[f\left(\tau,[u(\tau)-w(\tau)]^{*}\right)+p_{+}(\tau)\right] \mathrm{d} \tau \mid \\
& \leq \int_{0}^{1} \lambda\left|G\left(t_{1}, s\right)-G\left(t_{2}, s\right)\right| \mathrm{d} s \\
& \quad \times \int_{0}^{1} G(\tau, \tau)\left[f\left(\tau,[u(\tau)-w(\tau)]^{*}\right)+p_{+}(\tau)\right] \mathrm{d} \tau \\
& \leq \quad \lambda \int_{0}^{1}\left|G\left(t_{1}, s\right)-G\left(t_{2}, s\right)\right| \mathrm{d} s \cdot \max \left\{(L+1)^{\beta}, 1\right\} \\
& \quad \times \int_{0}^{1} G(\tau, \tau)\left[f(\tau, 1)+p_{+}(\tau)\right] \mathrm{d} \tau<\varepsilon .
\end{aligned}
$$

Therefore, $T(D)$ is equicontinuous on $[0,1]$. According to the Ascoli-Arzela Theorem, $T(D)$ is a relatively compact set.

At the end, Let $u_{n}(n=1,2,3, \ldots), u_{0} \in P, u_{n} \rightarrow u_{0}(n \rightarrow$ $+\infty)$. Then, $u_{n}$ is bounded, let $L_{2}=\sup \left\{\left\|u_{n}\right\|, n=0,1,2, \ldots\right\}$, for any $t \in[0,1]$, we have

$$
\left[u_{n}(t)-w(t)\right]^{*} \leq u_{n}(t) \leq\left\|u_{n}\right\| \leq L_{2} \leq L_{2}+1 .
$$

By (12), (34) and Lemma 3, we get

$$
f\left(t,\left[u_{n}(t)-w(t)\right]^{*}\right) \leq f\left(t, L_{2}+1\right) \leq\left(L_{2}+1\right)^{\beta} f(t, 1) .
$$

From (35), the continuity of $f$ and Lebesgue dominated convergence theorem, we have

$$
\begin{aligned}
& \left|T u_{n}(t)-T u_{0}(t)\right| \\
& =\lambda \int_{0}^{1} G(t, s) \mathrm{d} s \int_{0}^{1} G(s, \tau) \mid f\left(\tau,\left[u_{n}(\tau)-w(\tau)\right]^{*}\right) \\
& \quad-f\left(\tau,\left[u_{0}(\tau)-w(\tau)\right]^{*}\right) \mid \mathrm{d} \tau \\
& \leq \frac{1}{4} \lambda \int_{0}^{1} G(\tau, \tau) \mid f\left(\tau,\left[u_{n}(\tau)-w(\tau)\right]^{*}\right) \\
& -f\left(\tau,\left[u_{0}(\tau)-w(\tau)\right]^{*}\right) \mid \mathrm{d} \tau \longrightarrow 0 .
\end{aligned}
$$

Therefore, $T: P \rightarrow P$ is continuous. So $T: P \rightarrow P$ is a completely continuous operator.

The proof of our main result is based upon an application of the following fixed point theorem in a cone.

Theorem 6 (see [11]). Let $E$ be a Banach space, and $K \subset$ $E$ be a cone. Assume $\Omega_{1}, \Omega_{2}$ are open bounded subsets of $E$ 
with $0 \in \Omega_{1}, \bar{\Omega}_{1} \subset \Omega_{2}$, and let $T: K \cap\left(\bar{\Omega}_{2} \backslash \Omega_{1}\right) \rightarrow K$ be a completely continuous operator such that

(i) $\|T u\| \leq\|u\|, u \in K \cap \partial \Omega_{1}$, and $\|T u\| \geq\|u\|, u \in K \cap$ $\partial \Omega_{2} ;$ or

(ii) $\|T u\| \geq\|u\|, u \in K \cap \partial \Omega_{1}$, and $\|T u\| \leq\|u\|, u \in K \cap$ $\partial \Omega_{2}$.

Then, $T$ has at least one fixed point in $K \cap\left(\bar{\Omega}_{2} \backslash \Omega_{1}\right)$.

\section{The Main Results and Proofs}

Theorem 7. Suppose that $\left(H_{1}\right)$ and $\left(H_{2}\right)$ hold. Assume that there exist constants $0<\xi<\eta<1$ and $\Gamma$ such that

$$
\min _{t \in[\xi, \eta]} \frac{f(t, u)}{u} \geq \Gamma, \quad \text { if } u \geq r \xi(1-\eta)
$$

where

$$
\begin{gathered}
\Gamma=2\left[\lambda \xi(1-\eta)(\eta-\xi) \int_{0}^{1} G(\xi, s) A(s) d s\right]^{-1}, \\
A(s)=\min \{s(1-\eta), \xi(1-s)\} .
\end{gathered}
$$

Then, for $\lambda$ sufficiently small, BVP (1) has at least one positive solution $u \in C[0,1]$ for a.e. $t \in[0,1]$ in $P$.

Proof. Set

$$
\Omega_{d}=\{u \in P:\|u\|<d\}, \quad \partial \Omega_{d}=\{u \in P:\|u\|=d\} .
$$

Let

$$
\rho>r, \quad \lambda_{0}=\min \left\{1,4 \rho \cdot\left\{\left[(\max \{\rho, 1\})^{\beta}+1\right] l\right\}^{-1}\right\},
$$

where $r, l$ are defined in $\left(H_{2}\right)$. For any $u \in \partial \Omega_{\rho}$, we have $\|u\|=$ $\rho$, since

$$
\begin{aligned}
& w(t)=\int_{0}^{1} \mathrm{~d} s \int_{0}^{1} \lambda G(t, s) G(s, \tau) p_{-}(\tau) \mathrm{d} \tau \\
& \leq \frac{1}{4} \lambda G(t, t) \int_{0}^{1} p_{-}(\tau) \mathrm{d} \tau \\
& \leq \lambda r G(t, t), \quad t \in[0,1], \\
& \text { and } u(t) \geq G(t, t)\|u\| \geq r G(t, t),
\end{aligned}
$$

for any $\lambda \in\left(0, \lambda_{0}\right]$ and $u \in \partial \Omega_{\rho}$, we have

$$
u(t)-w(t) \geq r G(t, t)-\lambda r G(t, t) \geq 0, \quad t \in[0,1] .
$$

Noting that

$$
\begin{array}{r}
0 \leq u(t)-w(t) \leq u(t) \leq\|u\|=\rho \leq \max \{\rho, 1\}, \\
t \in[0,1] .
\end{array}
$$

From Lemma 3, we get

$$
\begin{aligned}
f\left(t,[u(t)-w(t)]^{*}\right) & \leq f(t, \max \{\rho, 1\}) \\
& \leq(\max \{\rho, 1\})^{\beta} f(t, 1) .
\end{aligned}
$$

Then, for any $\lambda \in\left(0, \lambda_{0}\right]$ and $u \in \partial \Omega_{\rho}$, we have

$T u(t)$

$$
\begin{gathered}
\int_{0}^{1} \mathrm{~d} s \int_{0}^{1} \lambda G(t, s) G(s, \tau)\left[f\left(\tau,[u(\tau)-w(\tau)]^{*}\right)\right. \\
\left.+p_{+}(\tau)\right] \mathrm{d} \tau \\
\leq \int_{0}^{1} \mathrm{~d} s \int_{0}^{1} \lambda G(t, t) G(\tau, \tau)\left[f\left(\tau,[u(\tau)-w(\tau)]^{*}\right)\right. \\
\left.+p_{+}(\tau)\right] \mathrm{d} \tau \\
\leq \frac{1}{4} \lambda_{0}\left[(\max \{\rho, 1\})^{\beta}+1\right] \int_{0}^{1} G(\tau, \tau)\left[f(\tau, 1)+p_{+}(\tau)\right] \mathrm{d} \tau \\
=\frac{1}{4} \lambda_{0}\left[(\max \{\rho, 1\})^{\beta}+1\right] l \leq \rho=\|u\| .
\end{gathered}
$$

Thus, we obtain that

$$
\|T u\| \leq\|u\|, \quad \forall u \in \partial \Omega_{\rho} .
$$

Let $R>\max \{\rho, 2 r\}$. For $t \in[\xi, \eta], u \in \partial \Omega_{R}$ and $\lambda \in\left(0, \lambda_{0}\right]$, we have

$$
\begin{aligned}
u(t)-w(t) & \geq R G(t, t)-\lambda r G(t, t) \geq R G(t, t)-\lambda \frac{R}{2} G(t, t) \\
& \geq R G(t, t)-\frac{R}{2} G(t, t)=\frac{R}{2} G(t, t) \geq \frac{R}{2} \xi(1-\eta) \\
& >r \xi(1-\eta)>0 .
\end{aligned}
$$

Hence, by (37) and (47), for any $u \in \partial \Omega_{R}$ and $\lambda \in\left(0, \lambda_{0}\right]$, we have

$$
\begin{aligned}
& T u(\xi)=\int_{0}^{1} \mathrm{~d} s \int_{0}^{1} \lambda G(\xi, s) G(s, \tau)\left[f\left(\tau,[u(\tau)-w(\tau)]^{*}\right)\right. \\
& \left.+p_{+}(\tau)\right] \mathrm{d} \tau \\
& \geq \int_{0}^{1} \mathrm{~d} s \int_{\xi}^{\eta} \lambda G(\xi, s) G(s, \tau)\left[f\left(\tau,[u(\tau)-w(\tau)]^{*}\right)\right. \\
& \left.+p_{+}(\tau)\right] \mathrm{d} \tau
\end{aligned}
$$




$$
\begin{aligned}
& \geq \int_{0}^{1} \lambda G(\xi, s) A(s) \mathrm{d} s \int_{\xi}^{\eta} f\left(\tau,[u(\tau)-w(\tau)]^{*}\right) \mathrm{d} \tau \\
& \geq \int_{0}^{1} \lambda G(\xi, s) A(s) \mathrm{d} s \cdot \Gamma \cdot \int_{\xi}^{\eta}[u(\tau)-w(\tau)] \mathrm{d} \tau \\
& \geq \int_{0}^{1} \lambda G(\xi, s) A(s) \mathrm{d} s \cdot \Gamma \cdot \frac{R}{2} \xi(1-\eta)(\eta-\xi) \\
& =R=\|u\| .
\end{aligned}
$$

Thus, we get

$$
\|T u\| \geq T u(\xi) \geq\|u\|, \quad \forall u \in \partial \Omega_{R}
$$

By Theorem 6, we know that $T$ has at least a fixed point $u^{*} \in \bar{\Omega}_{R} \backslash \Omega_{\rho}$ with $\left\|u^{*}\right\| \geq \rho$.

Thus, for any $\lambda \in\left(0, \lambda_{0}\right]$, we have

$$
\begin{aligned}
u^{*}(t)-w(t) & \geq G(t, t)\left\|u^{*}\right\|-\lambda G(t, t) \int_{0}^{1} p_{-}(\tau) \mathrm{d} \tau \\
& \geq \rho G(t, t)-\lambda r G(t, t) \\
& \geq \rho G(t, t)-\lambda r G(t, t) \geq 0, \quad t \in[0,1] .
\end{aligned}
$$

It follows from Lemma 4 that $u^{*}-w$ is a positive solution of BVP (1).

Corollary 8. Suppose that $\left(H_{1}\right)$ and $\left(H_{2}\right)$ hold. Assume that there exist constants $0<\xi_{1}<\eta_{1}<1$ and $\Gamma$ such that

$$
\lim _{u \rightarrow+\infty} \min _{t \in\left[\xi_{1}, \eta_{1}\right]} \frac{f(t, u)}{u}=+\infty \text {, }
$$

Then, for $\lambda$ adequately small, BVP (1) has at least one positive solution $u \in C[0,1]$ for a.e. $t \in[0,1]$ in $P$.

Proof. Obviously, (51) implies that (37) is satisfied. Thus, by Theorem 7, we know that Corollary 8 holds.

\section{An Example}

Now, we present an example to illustrate the main result.

Example 1. Consider the following BVP

$$
\begin{gathered}
u^{(4)}(t)-\lambda\left[t(1-t) u^{3 / 2}+\frac{1}{2}\left(\frac{1}{\sqrt{1-t}}-\frac{1}{\sqrt{t}}\right)\right]=0, \\
\text { a.e. } t \in(0,1), \\
u(0)=u(1)=u^{\prime \prime}(0)=u^{\prime \prime}(1)=0,
\end{gathered}
$$

where $\lambda$ is positive parameter, clearly

$$
f(t, u)=t(1-t) u^{3 / 2}, \quad p(t)=\frac{1}{2}\left(\frac{1}{\sqrt{1-t}}-\frac{1}{\sqrt{t}}\right) \text {. }
$$

Let

$$
\alpha=\frac{1}{2}, \quad \beta=2,
$$

then $\left(H_{1}\right)$ holds. By calculating, it is easy to obtain that

$$
\begin{gathered}
r=\int_{0}^{1} p_{-}(s) \mathrm{d} s=\sqrt{2}-1, \\
l=\int_{0}^{1} G(s, s)\left[f(s, 1)+p_{+}(s)\right] \mathrm{d} s=\frac{7}{60}(\sqrt{2}-1),
\end{gathered}
$$

thus $\left(H_{2}\right)$ holds. Obviously, for any fixed $\theta \in(0,1 / 2)$, we have

$$
\lim _{u \rightarrow+\infty} \min _{t \in[\theta, 1-\theta]} \frac{f(t, u)}{u}=+\infty .
$$

Let

$$
\rho=2>r
$$

then

$$
\lambda_{0}=\min \left\{1,4 \rho \cdot\left\{\left[(\max \{\rho, 1\})^{\beta}+1\right] l\right\}^{-1}\right\}=1,
$$

for any $\lambda \in\left(0, \lambda_{0}\right]$. By Corollary 8 , we know that BVP (52) has at least one positive solution $u \in C[0,1]$ for a.e. $t \in[0,1]$ in $P$.

\section{Acknowlegdments}

This paper is supported by the NNSF of China (10971045) and HEBNSF of China (A2012506010).

\section{References}

[1] S. P. Timoshenko, Theory of Elastic Stability, McGraw-Hill, New York, NY, USA, 1961.

[2] W. Soedel, Vibrations of Shells and Plates, Marcel Dekker, New York, NY, USA, 1993.

[3] E. Dulcska, "Soil settlement effects on buildings," in Developments in Geotechnical Engineering, vol. 69, Elsevier, Amsterdam, The Netherlands, 1992.

[4] D. R. Anderson and J. M. Davis, "Multiple solutions and eigenvalues for third-order right focal boundary value problems," Journal of Mathematical Analysis and Applications, vol. 267, no. 1, pp. 135-157, 2002.

[5] J. V. Baxley and L. J. Haywood, "Nonlinear boundary value problems with multiple solutions," Nonlinear Analysis: Theory, Methods \& Applications, vol. 47, no. 2, pp. 1187-1198, 2001.

[6] Z. Hao, L. Liu, and L. Debnath, "A necessary and sufficient condition for the existence of positive solutions of fourthorder singular boundary value problems," Applied Mathematics Letters, vol. 16, no. 3, pp. 279-285, 2003.

[7] R. P. Agarwal, Focal Boundary Value Problems for Differential and Difference Equations, vol. 436 of Mathematics and Its Applications, Kluwer Academic Publishers, Dordrecht, The Netherlands, 1998.

[8] R. P. Agarwal, D. O. Regan, and P. J. Y. Wong, Positive Solutions of Differential, Difference and Integral Equations, Kluwer Academic Publishers, Dordrecht, The Netherlands, 1999. 
[9] Z. Bai and H. Wang, "On positive solutions of some nonlinear fourth-order beam equations," Journal of Mathematical Analysis and Applications, vol. 270, no. 2, pp. 357-368, 2002.

[10] M. Feng and W. Ge, "Existence of positive solutions for singular eigenvalue problems," Electronic Journal of Differential Equations, vol. 105, pp. 1-9, 2006.

[11] D. J. Guo and V. Lakshmikantham, Nonlinear Problems in Abstract Cones, vol. 5 of Notes and Reports in Mathematics in Science and Engineering, Academic Press, Orlando, Fla, USA, 1988.

[12] J. M. Davis, P. W. Eloe, and J. Henderson, "Triple positive solutions and dependence on higher order derivatives," Journal of Mathematical Analysis and Applications, vol. 237, no. 2, pp. 710-720, 1999.

[13] J. M. Davis, J. Henderson, and P. J. Y. Wong, "General Lidstone problems: multiplicity and symmetry of solutions," Journal of Mathematical Analysis and Applications, vol. 251, no. 2, pp. 527548, 2000.

[14] J. R. Graef, C. Qian, and B. Yang, "Multiple symmetric positive solutions of a class of boundary value problems for higher order ordinary differential equations," Proceedings of the American Mathematical Society, vol. 131, no. 2, pp. 577-585, 2003.

[15] Y. Li, "Positive solutions of fourth-order periodic boundary value problems," Nonlinear Analysis: Theory, Methods \& Applications, vol. 54, no. 6, pp. 1069-1078, 2003.

[16] Y. Li, "Positive solutions of fourth-order boundary value problems with two parameters," Journal of Mathematical Analysis and Applications, vol. 281, no. 2, pp. 477-484, 2003.

[17] B. Liu, "Positive solutions of fourth-order two point boundary value problems," Applied Mathematics and Computation, vol. 148, no. 2, pp. 407-420, 2004.

[18] F. Li, Q. Zhang, and Z. Liang, "Existence and multiplicity of solutions of a kind of fourth-order boundary value problem," Nonlinear Analysis: Theory, Methods \& Applications, vol. 62, no. 5, pp. 803-816, 2005. 


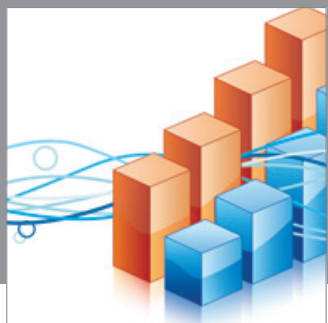

Advances in

Operations Research

mansans

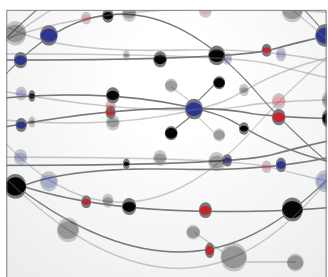

The Scientific World Journal
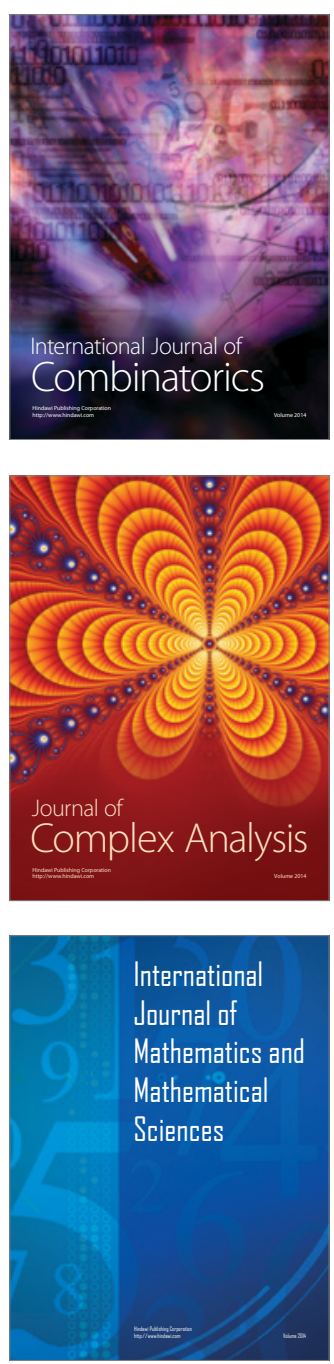
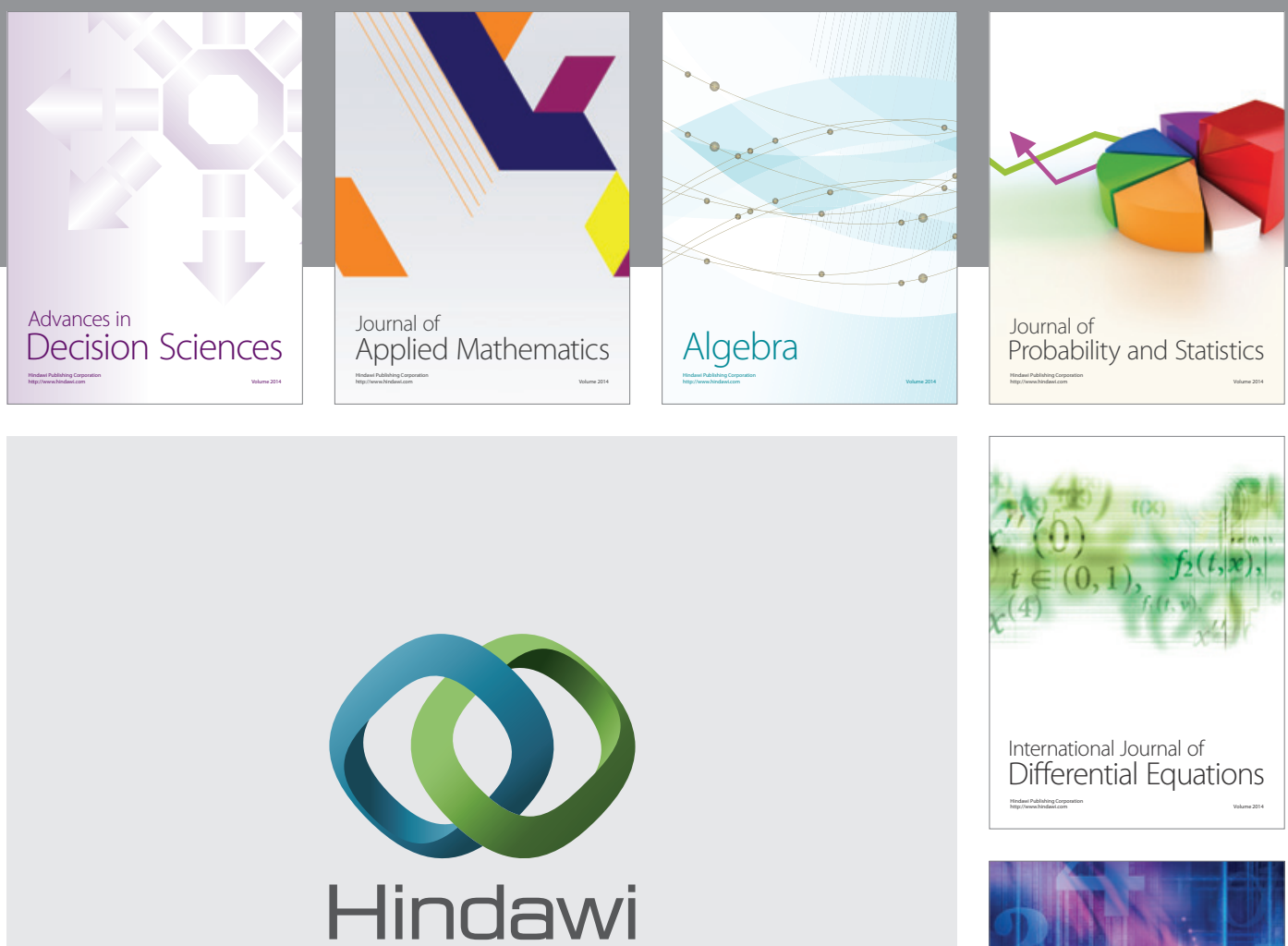

Submit your manuscripts at http://www.hindawi.com
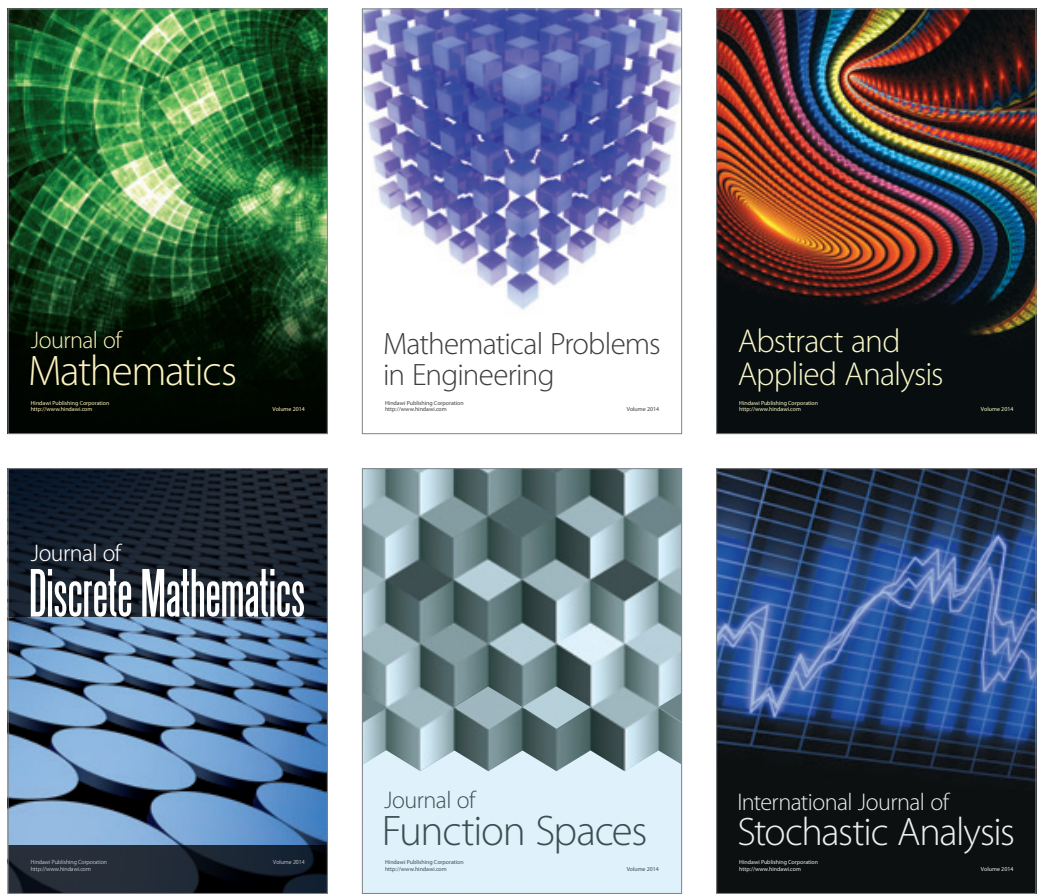

Journal of

Function Spaces

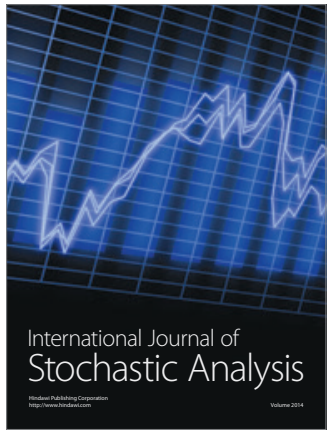

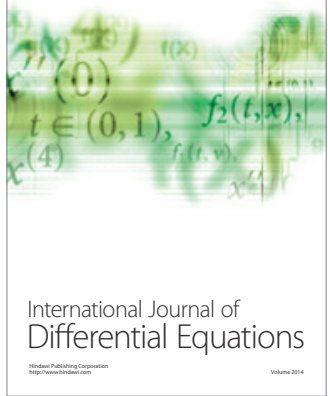
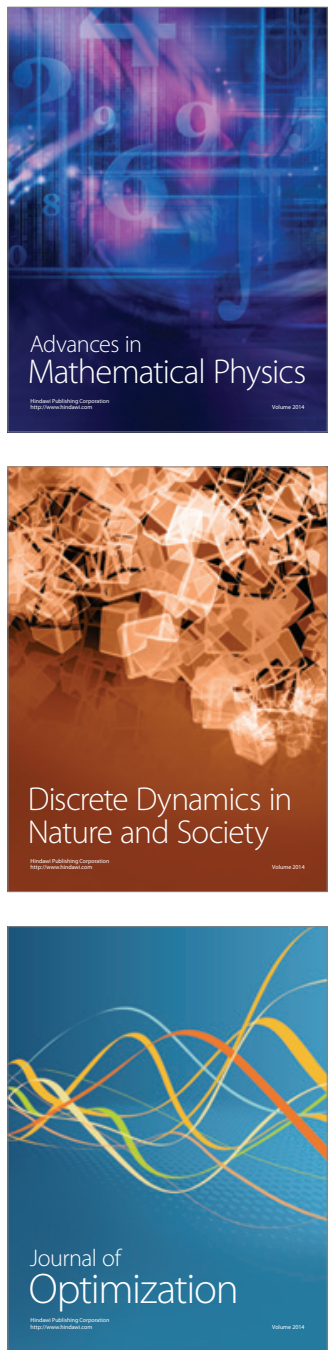